\title{
Evaluation of National Injury Surveillance Centre, India, 2015-16
}

Naveen Kumar Rastogi ${ }^{1}$, Kapil Goel ${ }^{2}$, Tanu Jain ${ }^{3}$, Samir Sodha ${ }^{4}$, Rajesh Yadav ${ }^{5}$, Chandra Shekhar Aggarwal ${ }^{6}$, Akshay Dhariwal ${ }^{7}$

${ }^{1}$ Epidemic Intelligence Service (EIS) Officer, National Centre for Disease Control, Delhi, India; ${ }^{2}$ Assistant Professor Epidemiology, Department of Community Medicine \& School of Public Health, Post Graduate Institute of Medical Education and Research (PGIMER), Chandigarh, India; ${ }^{3}$ Assistant Director General, Directorate General of Health Services, Ministry of Health \& Family Welfare, Government of India, New Delhi; ${ }^{4}$ Resident Advisor, India Epidemic Intelligence Service Program, Centers for Disease Control and Prevention (CDC), New Delhi, India; ${ }^{5}$ Public Health Specialist, Global Disease Detection Centre, Division of Global Health protection, Centers for Disease Control and Prevention (CDC), New Delhi, India; ${ }^{6} \mathrm{Head}$, Department of Epidemiology, National Centre for Disease Control, Delhi, India; ${ }^{7}$ Director, National Centre for Disease Control, Delhi, India

\begin{tabular}{|c|c|c|c|c|c|c|c|}
\hline Abstract & Introduction & Methodology & Results & Conclusion & References & Citation & Tables / Figures \\
\hline
\end{tabular}

\section{Corresponding Author}

Dr. Kapil Goel, Assistant Professor - Epidemiology, Dept. of Community Medicine \& School of Public Health,

Post Graduate Institute of Medical Education and Research (PGIMER), Chandigarh- 160012

E Mail ID: drkapil123@gmail.com

\section{Citation}

Rastogi NK, Goel K, Jain T, Sodha S, Yadav R, Aggarwal CS, Dhariwal A. Evaluation of National Injury Surveillance Centre, India, 2015-16. Indian J Comm Health. 2020;32(1):51-56.

Source of Funding: Nil Conflict of Interest: None declared

\section{Article Cycle}

Received: 14/11/2019; Revision: 20/12/2020; Accepted:15/02/2020; Published:31/03/2020

This work is licensed under a Creative Commons Attribution 4.0 International License.

\section{Abstract}

Background: Globally, injuries accounts for $9 \%$ of all deaths, but India account for $11 \%$. Due to limited data on injury characteristics, National Injury Surveillance Centre (NISC) was established in 2014 in New Delhi. Aim \& Objectives: To evaluate attributes of NISC and make evidence-based recommendations. Methods and Material: We conducted cross-sectional study and used US Centers for Disease Control and Prevention guidelines to assess simplicity, flexibility, acceptability, stability, timeliness, representativeness, usefulness, and data quality. We reviewed 2015 records and interviewed 20 key-informants. We used Epi-Info7 for analysis. Results: NISC captured 4043 injuries in 2015 from one hospital. Among five data entry operators, four reported lengthy format, but all reported it easy. Among ten relevant key-informants, all reported datamanagement software easy. System demonstrated flexibility in three variables. All 20 staff reported willingness to participate, and $90 \%$ felt quarterly reporting acceptable. Regarding stability, data was collected for $361 / 365$ days. Quarterly reports were available but only submitted annually. Regarding usefulness, all WHO-recommended variables included. Regarding data quality, $17 \%$ data-fields were missing. Conclusion: NISC is simple, flexible, stable, acceptable and potentially useful based on data captured. Timeliness based on annual reporting is high, can be improved to quarterly. We recommend training to improve data quality and integration of additional hospitals to improve representativeness.

\section{Keywords}

Injury; Surveillance; Data; Accuracy; Timeliness; Management; India.

\section{Introduction}

Globally in 2015, there were an estimated five million deaths from injuries, accounting for $9 \%$ of all deaths. WHO estimates that approximately $29 \%$ of global injury deaths are from intentional injuries: suicide, homicide and war; remaining $71 \%$ are unintentional injuries such as road traffic injuries, falls, drowning, burns, and poisoning.(1) In India, there were an estimated one million deaths from injuries in 2015, accounting for $11 \%$ of all deaths. WHO estimates that approximately $25 \%$ of injury deaths in India are from intentional injuries; remaining $75 \%$ are unintentional injuries.(1) Unintentional injuries are among the leading causes of mortality and morbidity in India. The problem is hidden and under recognized due to limited surveillance for injuries. In India most injuryrelated data comes from the National Crime Record Bureau, a source for mortality data but lacking information about pre hospital care, hospital care and risk factors. The National Injury Surveillance Centre (NISC) was established in Dr. Ram Manohar Lohia (RML) Hospital, New Delhi in 2014 by the Government of India to provide 
more informative data about injuries to guide policy formation and prevention intervention.

\section{Aims \& Objectives}

1. Describe and evaluate injury surveillance at RML Hospital to identify strengths and weaknesses.

2. Make evidence-based recommendations for improvement.

\section{Material \& Methods}

Study Type: A cross-sectional descriptive study. Study Area: NISC, Ram Manohar Lohia (RML) Hospital, New Delhi. Study Duration: We collected data between December 2015 and January 2016 from NISC.

Inclusion Criteria: All injuries reported in the casualty room from 1 January 2015 to 31 December 2015.

Exclusion Criteria: Some injuries directly reported and managed in outpatient department were not captured in this surveillance system.

Strategy for collection:

a. Surveillance description: To describe the surveillance system, we reviewed the available documents including data collection tool, documents for software development, the first annual report 2014-2015 of NISC, and the Memorandum of Expenditure Finance Committee (EFC) for the source of funding.(2) We also interviewed all staff of NISC including the program coordinator.

b. Surveillance evaluation: We designed our evaluation based on United States Centers for Disease Control and Prevention's Updated Guidelines for Evaluating Public Health Surveillance Systems (3) to assess qualitative and quantitative attributes: simplicity, flexibility, acceptability, stability, timeliness, representativeness, data quality, and usefulness. To assess these attributes we interviewed key informants from NISC and Trauma program team in Ministry of Health and Family Welfare with a semi structured questionnaire. We also reviewed the available annual/quarterly reports of NISC and analyzed the data maintained in Microsoft Excel.

Working Definition: Simplicity was evaluated based on ease and time of data collection, data entry, and data management. We assessed flexibility of the system to change as needed based on history of changes in variables and database. For acceptability, we evaluated willingness of staff to participate in the surveillance system. For stability, we looked for evidence in the system maintaining daily surveillance. We assessed usefulness based on inclusion of WHO recommended variables for injury surveillance, including identifier, age, sex, intent, place of occurrence, activity, nature of injury, mechanism of injury, adverse risk factors, and protective measures.(4) Data quality was assessed by the proportion of total missing, not known, or other specified data field, out of the total relevant data fields. We assessed the proportion of missing data in all 49 relevant variables, out of total 50 variables (excluding "remarks"). The 'not known' option was available in 18 variables, and the 'other' option specified in 16 variables. Proportion of misclassification was also seen among answers for these variables.

We conducted key informant interviews using a semistructured questionnaire with 10 contractual employees and 10 supporting permanent staff of NISC, Dr. Ram Manohar Lohia hospital, New Delhi (Table 1). Among 20 employees interviewed, 17 were working in NISC and three were working in the Trauma Program team in the Ministry of Health and Family Welfare. Only relevant key informants were interviewed for specific questions. Pilot testing of the questionnaire was done on staff nurse incharge. Duration for each interview was about 20-30 minutes. All interviews were done in person by the principle investigator.

Ethical Approval: Ethical approval was first obtained from the Institutional Ethical Committee.

Data Analysis: Data was entered and analyzed in Epi Info 7.1.5.2 to calculate proportions.

\section{Results}

Description of National Injury Surveillance Centre (NISC) NISC is situated in Dr RML hospital, New Delhi and is funded under the Indian National 12th Year plan (1st April 2012 -31st March 2017) under the "National Trauma Care Program". Funding comes directly from the Ministry of Health and Family Welfare.

Guidelines made by World Health Organization were used to design the data collection tool to capture demographic details, date of injury, place of injury, mechanism of injury, severity of injury, nature of injury, pre hospital care, treatment, outcome, and risk factors.

When injury victims come to the casualty or emergency room, they are first registered at the registration counter where data collection begins. Then a quick assessment is done and treatment is given in the casualty room. If the patient gets transferred or admitted to a particular ward, additional data are collected. When the patient is discharged from the particular ward or directly from casualty, final follow up data are collected which includes outcome in the form of recovered and improved, not recovered, residual disability, dead, or not known. Data formats are compiled at registration. This data collection is done in a separate comprehensive data capture format, other than the usual required data medical record file. After entry, data are analyzed by the injury surveillance team at RML. A final report is disseminated to the Burn and Trauma Programme Division in Ministry of Health and Family Welfare quarterly and annually (Figure 1).

Data collection started in July 2014. Data collections are done by the data entry operator daily and data management (transfer, entry, editing, storage, and backup of data) is done by a statistical assistant. Data analysis and dissemination is done by data analyst and biostatistician quarterly and annually.

Among 4043 injuries in 2015 at RML Hospital, 1607 (40\%) were due to fall, 1582 (39\%) from road traffic crash, 276 
(7\%) from assault, 178 (4\%) from stab/cut, 174 (4\%) burns, 91 (2\%) from fall of objects, $42(1 \%)$ from poisoning, and remaining 93 (2\%) injuries includes firearms, drowning, hanging, chocking, sport, animal bites etc. Self harm was reported in 52 (1\%). Among 1607 falls, 68\% were male and median age was 25 years (range: $0.1-100$ years). Most falls occurred at home (68\%). Among 1582 road traffic crash, $85 \%$ were male and median age was 30 (range: $0.4-92$ years). Most commonly affected were motorcycles riders (56\%) including $16 \%$ pedestrians and $7 \%$ three-wheeled vehicles riders/occupants. Among motorcycles riders, $87 \%$ reportedly wore helmets at the time of road traffic crash. Among those in four-wheel vehicles, $64 \%$ wore seat belts. Among 276 assault victims, $80 \%$ were male and median age was 30 (range: 1-82 years). No object was used in $46 \%$ of assaults and a stick/blunt object was used in 35\%. Among 174 burns, 61\% were male and median age was 22 (Range: 0.5-92 years). Most burns occurred at home (79\%).

\section{Evaluation of Attributes}

Simplicity: Follow up is required to complete data collection in two to three steps, first at emergency followed at the ward and finally at discharge of the patient. Home visit was not required to complete the data capture format. Among five data entry operators, four reported the format to be lengthy, but all 5 reported it to be easy to fill. Among 10 relevant key informants, all reported data management software to be easy and seven reported data management to be not time consuming.

Flexibility: Initially NISC used Microsoft Excel software for data entry, but in September 2016 an online server with a website was launched. Data entry operators were now able to enter data directly to the website. In March 2015, the surveillance system modified three variables (education, marital status, and gender) and added two new variables -mobile number and aadhaar number (a unique identification number provided by government of India to all residents).

Acceptability: All 20 staff reported willingness to participate in the surveillance system. The system is able to protect privacy and confidentiality by securing the data through user-id and password for staff, and $90 \%$ of key informants felt quarterly reporting was acceptable.

Stability: The surveillance system collects, manages, and provides data regularly without failure. Out of 365 days in 2015, data were collected for 361 days (99\%). No computer failures were reported to affect data collection, entry, or analysis.

Timeliness: From July 2014 the data were being entered daily. The annual report for July 2014 to July 2015 was disseminated before December 2015 when this evaluation began. The quarterly reports for January to March 2015 and April to June 2015 were not released, but for July to September 2015 and October to December 2015, reports were released within a month.
Representativeness: This surveillance system represents only casualty patients in this one government tertiary hospital in Delhi. There is more of an urban representation and it does not reflect the patient populations that would report to private hospitals.

Usefulness: All WHO recommended variables of core minimum data set are being collected including adverse measures like alcohol consumption or psychoactive substance abuse and protective measures like seat belt and helmet usage. All the information about risk factors was shared with the relevant stakeholders.

Data Quality: From 4043 injuries, total 198,107 data fields were entered in record. Data were missing for $17 \%$ of fields, particularly related to ICD-10 (100\%), total body surface area burned (76\%), and Glasgow Coma Scale (36\%) (Table 2).

\section{Discussion}

NISC at RML hospital is the first Indian public health surveillance system for injuries to be started by the Indian government as part of a plan for national sentinel surveillance. The surveillance system rates high in usefulness, simplicity, flexibility, stability, acceptability, and timeliness, but still needs improvement in representativeness and data quality (Table 3 ).

However, the system is relatively new, and data quality and representativeness is low, this data can be used for simply routine feedback and less important purposes instead of using for policy purposes. The use of robust injury surveillance data in future will help provide evidence based interventions to reduce morbidity and mortality due to injuries in India. In Australia, after implementing a strong road safety programme from about 1970 and injury surveillance in 1980s, national injury prevention policies were implemented based on the surveillance data. Deaths in male population due to external causes of injury and poisoning decreased from 80 per 100,000 in 1970 to 60 per 100,000 in 2000.(5)

For improving usefulness of this injury surveillance system in India, data quality will need to improve. In this system we found low data quality assessed by proportion of missing data, proportion of not known, and proportion of other specified. Similar challenges have been found elsewhere. In China, an evaluation of injury surveillance in an emergency department found rates of incomplete forms ranging from $24 \%$ to $50 \%$ throughout the various six hour intervals of the day.(6) We also were unable to find outcome of all injuries at RML because we could not follow patients easily and data entry operators could not capture complete data for outcome. ICD-10 coding was not being utilized by the surveillance system. In most countries of South East Asia and Western Pacific region like Australia, Korea, Maldives, Thailand and Vietnam, ICD10 coding has been feasible and maintained for injury surveillance to help standardize and improve data quality. 
(5) Data quality can be increased at RML by training and sensitization of the data entry operators and by appointing the trauma nurses for data collection rather than using data entry operators. A rating criterion was developed for measuring the various data characteristics for the evaluation of injury surveillance system in Australia.(7) Similar systematic approaches to assessing data quality can be considered in the future in India as more data become available.

This surveillance system was started as a pilot project in RML hospital in Delhi, India and is only representative of inpatients through casualty in this one government hospital. Representativeness will increase when other hospitals of Delhi and other states are integrated as per the plan of Government of India. Other countries of South East Asia and Western Pacific region like Australia, Bangladesh, China, Cambodia, Korea, Maldives, Myanmar, Thailand and Vietnam have started injury surveillance systems from multiple sentinel sites or through community surveillance systems.(6) In Egypt a National Injury surveillance system has been established constituting $55 \%$ of Egyptian health facilities which provides detailed and timely information including outpatient injury cases.(8) In India, other hospitals have also started injury surveillance as sentinel sites. All India Institute of Medical Sciences, New Delhi in collaboration with the National Trauma Research Institute, Australia has developed trauma registries in four sites: two in Delhi, one in Mumbai and one in Ahmedabad.(9) The National Institute of Mental Health and Neuro Sciences, Bangalore has also established injury surveillance in collaboration with WHO in Tumkur District, Karnataka, India.(10) Future efforts toward collaborating with these existing injury surveillance systems in India can facilitate plans for expansion and improved representativeness.

\section{Conclusion}

NISC is simple, flexible, acceptable, stable and potentially useful based on data captured. Timeliness based on annual reporting is high but representativeness and data quality is low.

\section{Recommendation}

We recommend continued vigilant efforts toward improvements in injury surveillance in India. Injury surveillance is relatively new in India but critically important to guide public policy for improvements in public health. To improve the data quality it is essential to evaluate the missing data, not known and other specified fields on regular interval. This could be part of quarterly or monthly analysis, so that immediate corrective action can be taken. Even blank fields can be reviewed at the time of discharge of patients by the data entry operators. Evaluations such as this one are essential to identify gaps and to help guide incremental improvements.

\section{Limitation of the study}

This evaluation had limitations. We were not able to assess sensitivity because there was no denominator of injury data available from the medical record files. We also were unable to assess predictive value positive because we were unable to contact patients as well as limited access to medical record files retrospectively and also due to availability of only secondary data from NISC.

\section{Relevance of the study}

This is the first evaluation study for National Injury Surveillance Centre in India, which identified gaps in data quality and representativeness to strengthen the surveillance system.

\section{Authors Contribution}

NKR, KG and TJ conceived the study design and concepts. $S S, R Y, C S A$ and $A D$ contributed to the study design. NKR and KG did the literature search. KG, and TJ facilitated the field work and NKR did the field epidemiological work. Data was analysed by NKR and supervised by KG and TJ. NKR wrote the first draft of the report. NKR, KG and TJ wrote the full report and manuscript. Manuscript was edited and reviewed by KG, TJ, SS, RY, CSA and AD. KG is the Guarantor of the research. All authors discussed the results and contributed to revision of the final manuscript.

\section{Acknowledgement}

Authors would like to acknowledge Dr. L N Gupta (Head of Neurosurgery Department, Dr RML Hospital, New Delhi) and Dr. Shailja Sharma (Programme coordinator, Trauma care programme, Directorate General Health Services, New Delhi) for their contributions and support.

\section{References}

1. World Health Statistics 2015 report. World Health Organization page-60 [Accessed on 18/12/19]. Available from: http://www.who.int/gho/publications/world health_statistics/20 15/en/.

2. EFC Memorandum on Continuation of the scheme "Capacity Building for developing trauma care facilities in Govt. Hospitals on National highways" during 12th Five Year Plan;25-26.

3. Updated Guidelines for Evaluating Public Health Surveillance Systems. Recommendations from the Guidelines Working Group. MMWR. July 27, 2001 / Vol. 50 / No. RR-13.

4. World Health Organization, 2001, Injury Surveillance Guidelines. [Accessed on 19/12/19]. Available from: http://www.who.int/violence injury prevention/publications/sur veillance/surveillance guidelines/en/.

5. World Health Organization, 2012. Profile of Injury Surveillance Systems in selected Member States of the Asia-Pacific Region. [Accessed on 21/12/19]. Available from: http://apps.searo.who.int/pds docs/B4799.pdf.

6. X Liu, L Li, H Cui, X Liu, V W Jackson. Evaluation of an emergency department-based injury surveillance project in China using WHO guidelines. BMJ Injury Prevention 2009; 15 73-74 Published Online First: 03 Apr 2009. doi: 10. 1136/ip. 2009.021790. [Accessed on 18/12/19]. Available from: http://injuryprevention.bmi.com/content/15/2/105.

7. Rebecca J Mitchell, Ann Williamson and Rod $\mathrm{O}^{\prime}$ Connor. The Development of an evaluation framework for injury surveillance system, Sydney, Australia.BMC Public Health 2009, 9:260. 
INDIAN JOURNAL OF COMMUNITY HEALTH / VOL 32 / ISSUE NO 01 / JAN - MAR 2020 [Accessed on 25/01/20]. Available from: http://www.biomedcentral.com/1471-2458/9/260.

8. R Saad, H S Jaffar, M El Setouhy, J M Hirshon. Evaluation of the National Injury Surveillance System in Egypt. BMJ Injury Prevention 2010, Volume 16, Issue suppl 1. [Accessed on 18/12/19]. Available from:

http://injuryprevention.bmj.com/content/16/Suppl_1/A56.2.
[Injury Surveillance System...] | Rastogi NK et al

9. Australia-India Trauma Systems Collaboration. [Accessed on 18/12/19]. Available from:http://aitsc.org/programs/.

10. Jayaram A, Gururaj G, Rajanna MS, et al Findings of a injury surveillance programme done in a rural district setup in India Injury Prevention 2010;16:A262. [Accessed on 23/12/19]. Available from: http://injuryprevention.bmj.com/content/injuryprev/16/Suppl1/A $\underline{\text { 262.1.full.pdf }}$

\section{Tables}

TABLE 1 LIST OF KEY INFORMANTS, INJURY SURVEILLANCE SYSTEM EVALUATION, DR. RAM MANOHAR LOHIA HOSPITAL, NEW DELHI, INDIA - 2015

\begin{tabular}{|l|l|}
\hline Contractual (10) & Supporting permanent (10) \\
\hline Data entry operator-5 & Staff nurse in-charge-1 \\
\hline Computer programmer-2 & Injury surveillance team-6 \\
\hline Statistician-3 & Trauma programme team-3 \\
\hline
\end{tabular}

TABLE 2 DATA QUALITY INDICATORS: PROPORTION OF MISSING DATA, NOT KNOWN, AND MISCLASSIFICATION, INJURY SURVEILLANCE SYSTEM EVALUATION, DR. RAM MANOHAR LOHIA HOSPITAL, NEW DELHI, INDIA - 2015

\begin{tabular}{|l|r|r|r|}
\hline Data Quality Indicators & N & n & 25 \\
\hline Total missing, not known, or other specified data & $1,98,107$ & 50,046 & 100 \\
\hline Total missing data & & & \\
\hline ICD-10 & 4,043 & 4,043 & 76 \\
\hline Total body surface area burned & 174 & 133 & 36 \\
\hline Glasgow Coma Scale & 421 & 152 & 9 \\
\hline Total not known data among relevant variables & 54,045 & 5,084 & 22 \\
\hline Nature of injury & 3,195 & 709 & 16 \\
\hline Severity of injury & 4,026 & 664 & 20 \\
\hline Total other specified data among relevant variables & 57,694 & 11,027 & 65 \\
\hline First aid provider & 3,127 & 2,031 & 52 \\
\hline Type of crash & 3,127 & 1,768 & 46 \\
\hline Road user type & 3,400 & 1,574 & 44 \\
\hline Place of crash & 3,403 & 1,511 & \\
\hline Misclassification & & & 99 \\
\hline First aid provider & 905 & 892 & 100 \\
\hline Type of crash & 767 & 767 & 100 \\
\hline Road user type & 812 & 812 & 98 \\
\hline Place of crash & 837 & 821 & \\
\hline
\end{tabular}

TABLE 3 ATTRIBUTE RATING, INJURY SURVEILLANCE SYSTEM EVALUATION, DR. RAM MANOHAR LOHIA HOSPITAL, NEW DELHI, INDIA - 2015

\begin{tabular}{|c|c|c|}
\hline Attribute & Rating & Justification \\
\hline Simplicity & High & Easy data management, Follow up required \\
\hline Flexibility & High & Changes made to the system as needed \\
\hline Acceptability & High & Staff reported willingness to participate \\
\hline Stability & High & 99\% daily surveillance \\
\hline Timeliness & High & Timely data collection and reports disseminated annually \\
\hline Representativeness & Low & Only inpatients of one hospital in Delhi involved \\
\hline Usefulness & High & $\begin{array}{l}\text { Offers globally standardised data and includes } 100 \% \text { of variables } \\
\text { recommended by WHO }\end{array}$ \\
\hline $\begin{array}{l}\text { Data quality/ } \\
\text { Completeness }\end{array}$ & Low & $\begin{array}{l}\text { Over } 25 \% \text { of data missing, or mentioned under not known and other specified } \\
\text { categories }\end{array}$ \\
\hline
\end{tabular}




\section{Figures}

FIGURE 1 FLOW CHART, PATIENT AND DATA FLOW IN NISC, DR. RAM MANOHAR LOHIA HOSPITAL, NEW DELHI, INDIA - 2015

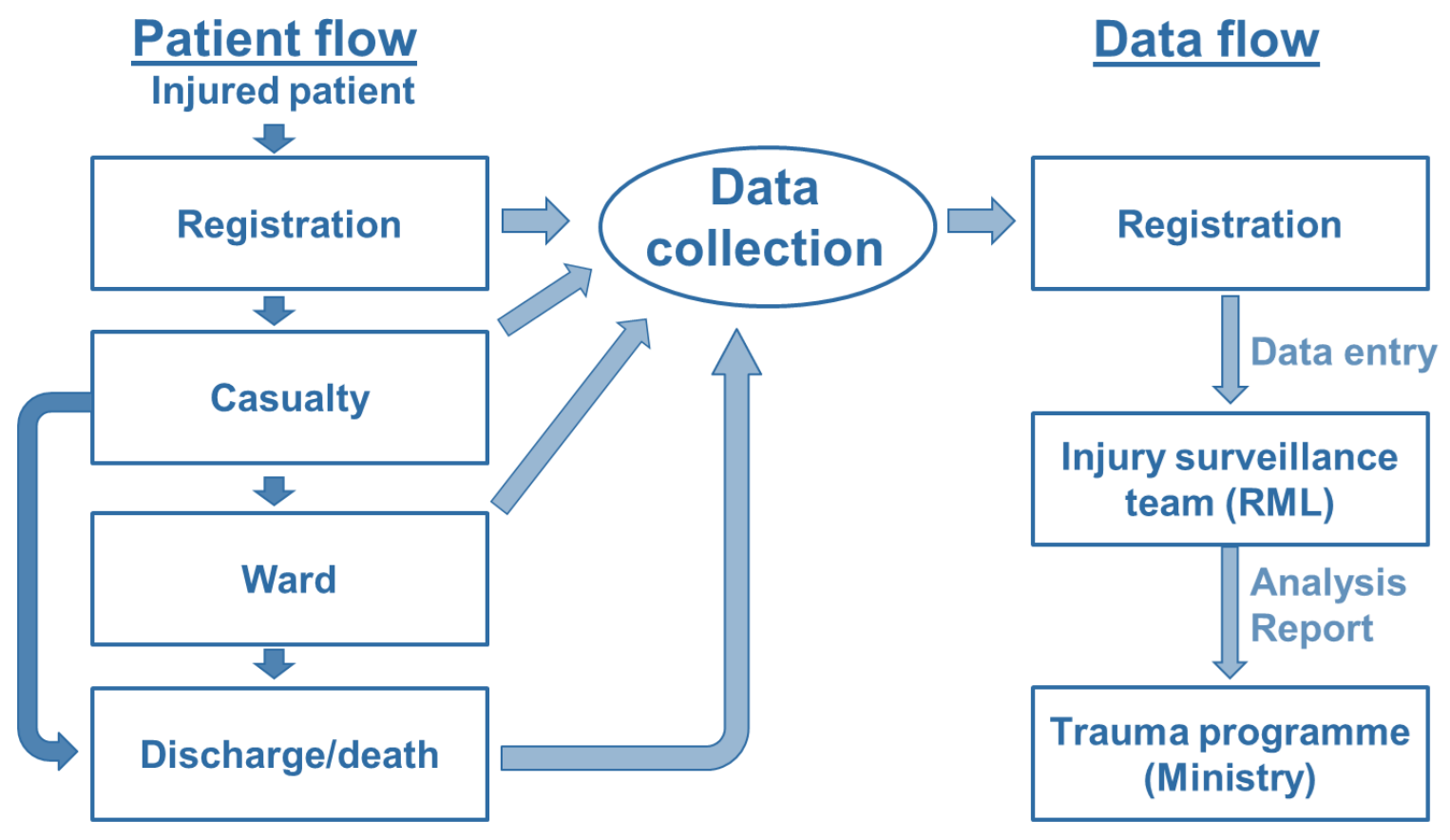

